\title{
Increased osteoclastogenesis in patients with vertebral fractures following discontinuation of denosumab treatment
}

\author{
Athanasios D Anastasilakis', Maria P Yavropoulou², Polyzois Makras³, Grigorios T Sakellariou4, \\ Fotini Papadopoulou ${ }^{5}$, Spyridon Gerou ${ }^{6}$ and Socrates E Papapoulos 7
}

${ }^{1}$ Department of Endocrinology, 424 General Military Hospital, Thessaloniki, Greece, ${ }^{2} 1$ st Department of Internal Medicine, Laboratory of Clinical and Molecular Endocrinology, AHEPA University Hospital, Aristotle University of Thessaloniki, Thessaloniki, Greece, ${ }^{3}$ Department of Endocrinology and Diabetes, 251 Hellenic Air Force \& VA General Hospital, Athens, Greece, ${ }^{4}$ Department of Rheumatology, 424 General Military Hospital, Thessaloniki, Greece, ${ }^{5}$ Private Practice, Thessaloniki, Greece, ${ }^{6}$ Laboratories 'Analysis', Thessaloniki, Greece, and ${ }^{7}$ Center for Bone Quality, Leiden University Medical Center, Leiden, The Netherlands

Correspondence should be addressed to A D Anastasilakis Email

a.anastasilakis@gmail.com

\begin{abstract}
Objective: To test the hypothesis that rebound of bone remodeling is responsible for clinical vertebral fractures reported in a few patients with osteoporosis after cessation of denosumab treatment.

Design: In this case-control study we compared clinical and biochemical characteristics of postmenopausal women with clinical vertebral fractures 8-16 months after the last injection of denosumab (Dmab/Fx+, $n=5)$ with those of treatment-naïve women with such fractures $(\mathrm{Fx}+, n=5)$. In addition, 5 women who discontinued denosumab treatment but did not sustain vertebral fractures 18-20 months after the last injection were studied $(D m a b / F x-, n=5)$. Methods: We measured serum microRNAs, gene expression of mRNAs of factors regulating formation and activity of osteoclasts and biochemical markers of bone and mineral metabolism. In Dmab/Fx+ and Fx+ women, blood was taken 4-8 weeks after the fracture.

Results: Compared to Fx+ women, Dmab/Fx+ women had higher serum P1NP and CTx levels, and significantly lower serum miR-503 and miR-222-2 that downregulate osteoclastogenesis and osteoclast activity, and higher RANK (13fold) and CTSK (2.6-fold) mRNA. The respective values of Dmab/Fx- women were in the same direction as those of Dmab/Fx+ women but of a lesser magnitude.

Conclusions: Bone fragility in women with clinical vertebral fractures after stopping denosumab therapy is pathophysiologically different from that of treatment-naïve women with osteoporosis and clinical vertebral fractures and it is associated with upregulation of markers of osteoclast formation and activity. The small number of women with this rare event studied is a limitation.
\end{abstract}

\section{Introduction}

Denosumab, a human monoclonal antibody that binds RANKL, is a potent inhibitor of the formation and activity of osteoclasts that reduces the risk of fractures in women with postmenopausal osteoporosis (1). In the 7-year extension of the pivotal 3-year placebo-controlled clinical trial, denosumab treatment increased BMD continuously at both the spine and the hip to levels within the range
() 2017 European Society of Endocrinology Printed in Great Britain
European Journal of Endocrinology

(2017) 176, 677-683 of osteopenia in a substantial number of patients $(2,3)$. In a number of such patients physicians decide to stop treatment due to the lower fracture risk. However, during the last year, clinical vertebral fractures, mostly multiple, were reported in a few women following discontinuation of denosumab treatment $(4,5,6,7,8)$ and raised questions about the appropriateness of such action (9). 
The mechanism responsible for the development of these fractures is unknown but it is hypothesized that they are due to the previously reported transient increase in bone turnover and rapid loss of BMD following cessation of denosumab (10). Some authors even proposed the term 'rebound-associated vertebral fractures' to characterize their pathophysiology (8) but direct evidence for this is lacking. In the present study we tested this hypothesis and we compared clinical and biochemical characteristics of patients with vertebral fractures following the discontinuation of denosumab treatment with those of treatment-naïve women with osteoporosis and recent clinical vertebral fractures. In addition, we investigated women without vertebral fractures after stopping denosumab to obtain more insight into the mechanism(s) underlying bone fragility.

\section{Patients and methods}

\section{Study design}

Case-control study of postmenopausal women with osteoporosis attending the outpatient clinic for Metabolic Bone Diseases of the 424 General Military Hospital. Cases were women who discontinued denosumab treatment and sustained clinical vertebral fractures 8-16 months after the last injection (Dmab/Fx+, $n=5)$; controls were treatment-naïve women with clinical vertebral fractures matched for age and time of blood sampling after the fractures to cases $(\mathrm{Fx}+, n=5)$. The primary objective of the study was to assess the potential differences in biochemical signals of osteoclast formation and activity between these two groups. In addition, five women of comparable age to cases, who discontinued denosumab treatment but did not sustain vertebral fractures $18-20$ months after the last injection, were also studied (Dmab/Fx-). In all women causes of secondary osteoporosis and/or fractures were excluded and none used any medications that could affect bone metabolism. The denosumab-treated women had never received any other treatment for osteoporosis. Informed consent was obtained from all patients and the study was approved by the local Ethics Committee.

\section{Methods}

A full medical history including a detailed history of denosumab use, time of its discontinuation and time of fracture, in those who had experienced a vertebral fracture, were obtained from all patients. Spine radiographs were performed in all patients and were evaluated for the presence, site, number and severity of vertebral fractures (11). Bone mineral density (BMD) was measured in all patients by DXA using a DPX-IQ densitometer (Lunar Corporation, Madison, WI, USA); in Dmab/Fx+ women, BMD was measured at the time of the last injection of denosumab while in treatmentnaive women it was measured at the time of the vertebral fracture. In Dmab/Fx- women BMD was measured before treatment, at treatment discontinuation as well as at the time of blood sampling. Morning fasting blood samples for the measurement of parameters of bone metabolism, microRNAs and gene analyses were obtained from all patients. In patients with vertebral fractures, either treatment-naïve or after discontinuation of denosumab, blood was obtained 4-8 weeks after the fracture.

Serum procollagen type $1 \mathrm{~N}$-terminal propeptide (P1NP) ,C-terminal telopeptide of type 1 collagen (CTx), intact parathyroid hormone (PTH), 25-hydroxyvitamin D (25-OHD), osteoprotegerin (OPG), soluble receptor activator of nuclear factor kappaB ligand (sRANKL) and sclerostin were measured as previously described (12).

\section{Serum microRNA and gene analyses}

To obtain a better insight of bone remodeling at the tissue level we analyzed the expression profile of specific microRNAs (miRs) and their related genes in the serum of patients. miRs are a class of naturally occurring small non-coding RNAs that are resistant to RNases and repress the expression of their target genes. They are present in serum and plasma of humans and are linked to biological function at tissue microenvironment in various diseases. There is evidence of a direct link between circulating miRs and altered bone metabolism. For example, Seeliger et al. (13) showed a robust association between the expression of certain miRs in serum and bone tissue in patients with osteoporosis and fractures and Weilner et al. reported that miRs in serum of patients with fractures can influence osteogenic differentiation (14). We analyzed in the serum of our patients the expression of 3 miRs (miR-21, miR503 and miR-222-2) and of mRNAs of their related genes that are involved in osteoclast formation and function (RANKL, RANK, cathepsin K (CTSK) and tartrate-resistant acid phosphatase (TRAP)) respectively $(15,16,17)$.

Total RNA was extracted from $200 \mu \mathrm{L}$ of serum sample using the miRNeasy Serum/Plasma Kit, according to the manufacturer's instructions (Qiagen). During the purification process a synthetic RNA sequence (spike ins: C. elegans miR-39) was added in appropriate amount to serum preparations after homogenization 
with the QIAzol lysis reagent to control for variations in recovery and amplification efficiency between RNA preparations. $1.25 \mathrm{mg} / \mathrm{mL}$ bacteriophage MS2 RNA was used as a carrier. The amount and purity of RNA was estimated by photometry (Thermo Scientific NanoDropTM 1000 Spectrophotometer). Subsequently, miRNA was transcribed to cDNA using the miScript II RT Kit (Qiagen) and one part of the total RNA was transcribed to firststrand cDNA using the QuantiNova Reverse Transcription Kit (Qiagen) for gene expression analysis.

Cycling was performed under standardized conditions with 2× QuantiTect SYBR Green PCR Master Mix on the QIAGEN Rotor-Gene Q (Corbett Rotor-Gene 6000) realtime PCR cycler. PCR was performed in triplicates.

A panel of 3 invariant microRNAs, two snoRNAs (SNORD95 and SNORD96A) and one snRNA (RNU6-2) were used to normalize the variability in sample loading and real-time RT-PCR efficiency. We analyzed the expression of miR-21-2, miR-503 and miR-222-2 sequences customized by Qiagen (miScript Primer Assays) and the expression of RANKL, RANK, CTSK and TRAP mRNAs. The sequences of both the forward and reverse primers were also customized by Qiagen (QuantiTect Primer Assays). The relative expression levels of each gene were determined by the cycle number via q-PCR, with their levels normalized to the glyceraldehyde 3-phosphate dehydrogenase (GADPH) cycle number using the $2^{\mathrm{DDCT}}$ method.

\section{Statistical analysis}

Data for continuous variables are presented as median \pm interquartile range (IQR). Using histograms and Q-Q plots, none of the continuous variables was found to be normally distributed. Therefore, Mann-Whitney test was used for comparison between groups in case of continuous variables. Spearman's correlation was used in the examination of the relationship between time from last injection and serum CTX values. A two-sided $P$-value of less than 0.05 was considered statistically significant. Statistical analysis was performed with SPSS for Windows, version 22.0 (IBM Corporation).

\section{Results}

There were no differences in age or BMI among the three groups of patients or in the number of fractures and time of blood sampling between the two groups with vertebral fractures (Table 1). Other characteristics of the study groups are also shown in Table 1 . Serum calcium and PTH levels and 25OHD concentrations were similar between the three groups $(10.2 \pm 1.4$ vs $9.7 \pm 1.1$ vs $9.6 \pm 0.7 \mathrm{mg} / \mathrm{dL}$; $50.1 \pm 15.9$ vs $47.2 \pm 31.6$ vs $53.1 \pm 23.9 \mathrm{pg} / \mathrm{mL} ; 16.3 \pm 6.2$ vs $18.2 \pm 7.3$ vs $19.5 \pm 9.7 \mathrm{ng} / \mathrm{mL} ; P>0.05$ for all).

\section{Patients with vertebral fractures}

Compared to treatment-naïve women, those with vertebral fractures after denosumab discontinuation had significantly higher lumbar spine BMD values $(P=0.029)$ while femoral neck BMD was not different between the two groups. All fractures in both groups were moderate or severe according to Genant classification (11) without differences between the groups and occurred at the lower thoracic and lumbar spine (T9-L5); two of the 5 Dmab/Fx+ women had also prevalent vertebral fractures. Compared to $\mathrm{Fx}+$ women, Dmab/Fx+ women had higher serum P1NP $(37.8 \pm 10.4$ vs $29.0 \pm 7.0 \mathrm{ng} / \mathrm{mL}, P=0.016)$ (Fig. 1A) and CTx levels $(534.0 \pm 271.0$ vs $401.0 \pm 185.5 \mathrm{pg} /$ $\mathrm{mL}, \quad P=0.095$ ) (Fig. 1B) and lower sclerostin levels $(22.36 \pm 19.15$ vs $46.94 \pm 9.39 \mathrm{pmol} / \mathrm{L}, P=0.008)$. Serum RANKL and OPG were not different between the two

Table 1 Characteristics of the study groups. Data are presented as median \pm IQR.

\begin{tabular}{l}
\hline \\
\hline Age (years) \\
BMI (kg/m²) \\
Time of blood sampling after Fx (weeks) \\
Number of Fxs \\
LS-BMD (T-score) \\
FN-BMD (T-score) \\
Duration of denosumab treatment (years) \\
Time from last denosumab injection (months)
\end{tabular}

\begin{tabular}{c}
\hline Dmab/Fx+ $(n=5)$ \\
\hline $71 \pm 21$ \\
$26.75 \pm 6.57$ \\
$5 \pm 3$ \\
$2 \pm 1.5$ \\
$-2.05 \pm 0.40$ \\
$-2.10 \pm 0.30$ \\
$3 \pm 1$ \\
$10 \pm 5$
\end{tabular}

$\mathbf{F x}+(n=5)$
$65 \pm 15$
$26.01 \pm 9.54$
$5 \pm 3$
$1 \pm 2$
$-3.10 \pm 1.30^{\mathrm{a}}$
$-2.60 \pm 0.50$
-
-
$69+5.5$ $32.84 \pm 5.50$

$-1.60 \pm 1.40^{\mathrm{b}}$

$-2.15 \pm 2.10$

$1 \pm 0.5^{\mathrm{a}}$ $19 \pm 4^{\mathrm{a}}$

a $P<0.05$ compared to the Dmab/Fx+ group; ${ }^{b} P<0.05$ compared to the $\mathrm{Fx}+$ group; BMD was measured at the time of denosumab discontinuation in the $\mathrm{Dmab} / \mathrm{Fx}+$ and $\mathrm{Dmab} / \mathrm{Fx}$ - groups and at the time of fracture in the $\mathrm{Fx}+$ group.

BMD, bone mineral density; BMI, body mass index; Dmab, denosumab; FN, femoral neck; Fx, vertebral fracture; LS, lumbar spine. 
groups $(0.12 \pm 0.06$ vs $0.20 \pm 0.12 \mathrm{pmol} / \mathrm{L}, P=0.222$ and $5.60 \pm 4.15$ vs $7.70 \pm 3.78 \mathrm{pmol} / \mathrm{L}, P=0.310$ respectively).

\section{Patients without vertebral fractures}

These patients had no prevalent vertebral fractures and did not sustain fractures after discontinuation of denosumab. Treatment significantly increased LS-BMD T-score by $11.0 \%$ from $-2.40 \pm 0.60$ at baseline to $-1.60 \pm 1.40$ at discontinuation; thereafter LS-BMD T-score decreased again toward baseline to $-2.20 \pm 0.80$ at the time of the present study (18-20 months after the last injection). Thus there was a rapid bone loss after stopping denosumab, as previously described. BMD at denosumab discontinuation was not different between Dmab/Fx+ and Dmab/Fxpatients, but a type two error cannot be excluded due to the small sample. In the latter patients, by design, time of blood sampling was significantly longer than in Dmab/ $\mathrm{Fx}+$ patients. Biochemical parameters of bone metabolism were not different between the two groups except for serum CTx values which were significantly higher in

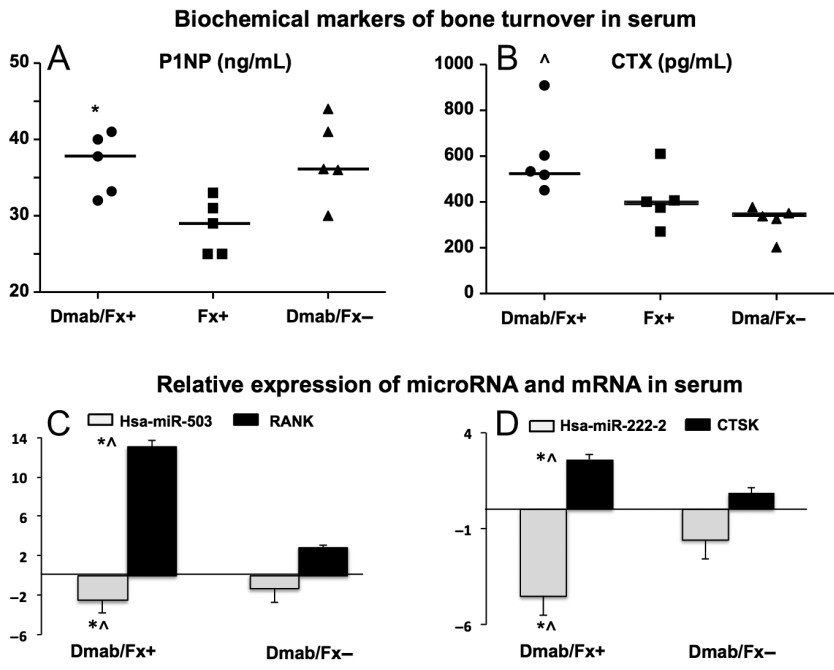

\section{Figure 1}

Serum levels of P1NP (A) and CTx (B) in the 3 three groups of studied women; medians are also shown. Differences in the expression of serum miR-503 and RANK mRNA (C) and miR-222-2 and cathepsin K mRNA (D) in Dmab/Fx+ and Dmab/ $\mathrm{Fx}$ - women relative to those of $\mathrm{Fx}+$ women.

$\star P<0.05$ compared to $\mathrm{Fx}+$ group, ${ }^{\wedge} P<0.05$ compared to Dmab/Fx- group. Dmab/Fx+, women with vertebral fractures after discontinuation of denosumab; Dmab/Fx-, women without vertebral fractures after discontinuation of denosumab; Fx+, treatment-naïve osteoporotic women with vertebral fractures.
Dmab/Fx+ patients $(534.0 \pm 271.0$ vs $338.0 \pm 100.5 \mathrm{pg} / \mathrm{mL}$, $P=0.008)$ (Fig. 1B).

\section{Expression of circulating microRNAs}

Compared to treatment-naïve women, the expression of miR-503 in serum was decreased both in Dmab/Fx+ and Dmab/Fx- women with the former group showing the highest and statistically significant decrease. These changes were associated with increases in RANK mRNA, which is negatively regulated by miR-503, by 13 -fold (Fig. 1C). Similarly, expression of miR-222-2 in serum was significantly lower in Dmab/Fx+ women while CTSK mRNA, which is negatively regulated by miR-222-2 at cellular level, increased with the highest expression (2.6fold increase) observed in Dmab/Fx+ women (Fig. 1D). Since miRs are secreted into the circulation from cells of various tissues reflecting local events, the reported changes are suggestive of a significant upregulation of osteoclast formation and osteoclast activity in women who received denosumab and sustained vertebral fractures after its discontinuation. However, the molecular mechanism(s) responsible for the observed changes of miRs following discontinuation of denosumab or the upstream regulation of miRs in general are ill-defined and there are no data available on miRs expression during or after any osteoporosis therapy for comparison. Serum levels of $R A N K L$ and OPG mRNA were marginally detectable and there were no differences among the three groups; there was no also difference between groups in TRAP mRNA levels (data not shown).

\section{Time of denosumab injection and bone resorption}

In all women who received denosumab (Dmab/Fx+ and $\mathrm{Dmab} / \mathrm{Fx}$ - combined) there was a significant negative correlation between the time of the last denosumab injection (range 8-24 months) and measured serum CTx levels (Spearman's $r=-0.827, P=0.008$ ).

\section{Discussion}

We present here the evidence of increased osteoclast formation and activity in denosumab-treated women with osteoporosis who sustained clinical vertebral fractures 8-16 months after the last injection of the monoclonal antibody. Interpretation of results of laboratory parameters, particularly bone turnover markers, following clinical fractures may be hampered 
by changes due to fracture healing rather than to mechanisms related to bone fragility. For this reason we studied treatment-naïve women with osteoporosis who presented with clinical vertebral fractures and we obtained blood at the same time following the fracture as in the denosumab-treated women. We show here that Dmab/Fx+ women had significant decreases in the expression of miRs that negatively regulate the osteoclast formation and activity in vitro $(15,16,17)$ associated with increases in the mRNA of their target genes RANK and GTSK in serum; in addition, had higher levels of bone turnover markers. These findings together imply the upregulation of osteoclastogenesis and osteoclast activity as a critical contributor to the increased vertebral fragility following the discontinuation of denosumab treatment.

Reversibility of the suppressive effect of denosumab on bone turnover has been previously demonstrated biochemically and histologically $(10,18)$ but has not been associated with increased bone fragility. Bone et al. reported no clinical vertebral fractures in 128 women followed for 2 years after the discontinuation of denosumab while Brown et al. (19) reported similar rates of fractures $\geq 7$ months after the last injection of denosumab or placebo. In a recent analysis of data from the FREEDOM study reported in abstract form, patients who discontinued denosumab treatment had a higher incidence of vertebral fractures relative to the on-treatment period but similar to that of women following discontinuation of placebo in the same study (20). However, a greater percentage of those who discontinued denosumab had multiple vertebral fractures. The authors recommended transition to another therapy of patients who discontinue denosumab after the 6-month dosing interval.

While our data confirm the hypothesis that upregulation of osteoclastogenesis and osteoclast activity contributes to the pathogenesis of vertebral fractures soon after cessation of denosumab treatment, they do not help in the identification of patients at highest risk for such fractures. In the above-mentioned study (20), the incidence of vertebral fractures following treatment cessation was $5.6 \%$ and prior vertebral fractures either at baseline or during treatment were the strongest predictors of off-treatment vertebral fractures, but indices of bone turnover were not measured. In our study, two of the five Dmab/Fx+ women had prevalent vertebral fractures as opposed to none in Dmab/Fx- women.

Dmab/Fx- women responded, as expected, in showing increases in BMD with treatment and a rapid decline to values close to baseline 19 months offtreatment. Laboratory indices of osteoclastogenesis and osteoclast activity, including CTx, were found between those of women with Dmab/Fx+ and those with $\mathrm{Fx}+$, a pattern that can be considered consistent with the timing of blood sampling. Whether the values attained in patients with fractures were consistently higher than in those without fractures cannot not be concluded due to the time of sampling. We chose not to take blood samples earlier because clinical vertebral fractures can develop up to 16 months after the last injection of denosumab and we thought that taking blood samples later will exclude the possibility of the event.

The kinetics of the marker of bone resorption, serum CTx, was described in detail by Bone et al. in patients treated with denosumab for 2 years followed for an additional 2 years off-treatment (10). Blood samples were obtained at 9 and 12 months after the last injection of denosumab and 6-monthly, thereafter. They showed a significant increase in serum CTx values above pretreatment values at 9 months with a peak at 12 months after the last injection and a progressive decrease thereafter. Our results, despite the small number of observations, conform to these findings in showing a significant correlation between the time since last denosumab injection and serum CTx values in all patients who were treated with denosumab independently of the presence of vertebral fractures.

Formation and activity of osteoclasts following cessation of denosumab treatment is a dynamic process that starts with the uninhibited, synchronous, rapid release of RANKL. However, this is transient, as shown here by the finding of normal serum RANKL and no changes in miR 21-2 and RANKL mRNA at a median 10 months after the last injection. This assumed initial increase in $R A N K L$ is responsible for the dramatic increase in osteoclastogenesis evidenced by the 13-fold increase in levels of RANKL mRNA followed by an increased activity of osteoclasts demonstrated by the upregulation of cathepsin $\mathrm{K}$ mRNA. These changes were transient as expected by the short half-life of osteoclasts and suggested by the kinetics of serum CTX values. Thus, while upregulation of osteoclastogenesis and osteoclast activity clearly differentiates patients with vertebral fractures following the discontinuation of denosumab from treatment-naïve patients with fractures, it does not help in predicting which patients will fracture after stopping denosumab. The rate of multiple vertebral fractures increased after stopping denosumab in the FREEDOM study but the absolute risk was low (3.4\%) (18). Thus, 
either these few patients have a difficult to explain, much higher upregulation of osteoclastogenesis or, more likely, they have microstructural bone changes predisposing to increased vertebral fragility when exposed to high osteoclast activity. The latter hypothesis is supported by the identification of prevalent vertebral fractures as the strongest risk factor after denosumab discontinuation in FREEDOM. Confirming or refuting either hypothesis needs additional data; however, that will be difficult to obtain due to the rarity of the event.

The main limitation of our study is the small number of women studied but the event is uncommon and only recently described. The strengths of our study include the use of a relevant control group and measurements of miRs and mRNAs of genes controlling osteoclastogenesis and osteoclast activity in the assessment of the response. The use of the latter, never reported previously during or after treatment with denosumab, helped to better define the response following treatment cessation and clarified the different mechanism of bone fragility in the two groups of patients with vertebral fractures.

\section{Declaration of interest}

Athanasios D Anastasilakis has received lecture fees from Amgen, Lilly, ITF Hellas, ELPEN and VIANEX. Polyzois Makras has received lecture fees and research grants from Amgen; lecture fees from Glaxo, Lilly, Pfizer, Leo, Genesis, ELPEN and VIANEX. Fotini Papadopoulou has received lecture fees from Lilly, ITF Hellas, Glaxo and VIANEX. Socrates E Papapoulos has received consulting/speaking fees from Amgen, Axsome, Merck, Mereo Biopharma and UCB; Maria Yavropoulou, Grigorios T Sakellariou and Spyridon Gerou have nothing to declare.

Funding

This research did not receive any specific grant from any funding agency in the public, commercial or not-for-profit sector.

\section{References}

1 Cummings SR, San Martin J, McClung MR, Siris ES, Eastell R, Reid IR, Delmas P, Zoog HB, Austin M, Wang A et al. Denosumab for prevention of fractures in postmenopausal women with osteoporosis. New England Journal of Medicine 2009361 756-765. (doi:10.1056/ NEJMoa0809493)

2 Papapoulos S, Lippuner K, Roux C, Lin CJ, Kendler DL, Lewiecki EM, Brandi ML, Czerwiński E, Franek E, Lakatos P et al. The effect of 8 or 5 years of denosumab treatment in postmenopausal women with osteoporosis: results from the FREEDOM Extension study. Osteoporosis International 201526 2773-2783. (doi:10.1007/s00198-015-3234-7)

3 Ferrari S, Libanati C, Lin CJ, Adami S, Brown JP, Cosman F, Czerwiński E, de Gregório LH, Malouf J, Reginster JY et al. Relationship between total hip BMD T-score and incidence of nonvertebral fracture with up to 8 years of denosumab treatment. Journal of Bone and Mineral Research 201530 (Supplement 1). (Available at: http://www.asbmr.org/education/ AbstractDetail?aid=11d861c9-68e4-4994-88e0-c6b94948e898). Accessed on 7 November 2016.

4 Anastasilakis AD \& Makras P. Multiple clinical vertebral fractures following denosumab discontinuation. Osteoporosis International 2016 27 1929-1930. (doi:10.1007/s00198-015-3459-5)

5 Aubry-Rozier B, Gonzalez-Rodriguez E, Stoll D \& Lamy O. Severe spontaneous vertebral fractures after denosumab discontinuation: three case reports. Osteoporosis International 201627 1923-1925. (doi:10.1007/s00198-015-3380-y)

6 Lamy O, Gonzalez-Rodriguez E, Stoll D, Hans D \& Aubry-Rozier B. Severe rebound-associated vertebral fractures after denosumab discontinuation: nine clinical cases report. Journal of Clinical Endocrinology and Metabolism 2017 102 354-358. (doi:10.1210/ jc.2016-3170)

7 Polyzos SA \& Terpos E. Clinical vertebral fractures following denosumab discontinuation. Endocrine 201654 271-272. (doi:10.1007/s12020-016-1030-6)

8 Popp AW, Zysset PK \& Lippuner K. Rebound-associated vertebral fractures after discontinuation of denosumab-from clinic and biomechanics. Osteoporosis International 201627 1917-1921. (doi:10.1007/s00198-015-3458-6)

9 McClung MR. Cancel the denosumab holiday. Osteoporosis International 201627 1677-1682. (doi:10.1007/s00198-016-3553-3)

10 Bone HG, Bolognese MA, Yuen CK, Kendler DL, Miller PD, Yang YC, Grazette L, San Martin J \& Gallagher JC. Effects of denosumab treatment and discontinuation on bone mineral density and bone turnover markers in postmenopausal women with low bone mass. Journal of Clinical Endocrinology and Metabolism 201196 972-980. (doi:10.1210/jc.2010-1502)

11 Genant HK, Jergas M, Palermo L, Nevitt M, Valentin RS, Black D \& Cummings SR. Comparison of semiquantitative visual and quantitative morphometric assessment of prevalent and incident vertebral fractures in osteoporosis The Study of Osteoporotic Fractures Research Group. Journal of Bone and Mineral Research 199611 984-996. (doi:10.1002/jbmr.5650110716)

12 Anastasilakis AD, Polyzos SA, Gkiomisi A, Saridakis ZG, Digkas D, Bisbinas I, Sakellariou GT, Papatheodorou A, Kokkoris P \& Makras P. Denosumab versus zoledronic acid in patients previously treated with zoledronic acid. Osteoporosis International 201526 2521-2527. (doi:10.1007/s00198-015-3174-2)

13 Seeliger C, Karpinski K, Haug AT, Vester H, Schmitt A, Bauer JS \& van Griensven M. Five freely circulating miRNAs and bone tissue miRNAs are associated with osteoporotic fractures. Journal of Bone and Mineral Research 201429 1718-1728. (doi:10.1002/jbmr.2175)

14 Weilner S, Skalicky S, Salzer B, Keider V, Wagner M, Hildner F, Gabriel C, Dovjak P, Pietschmann P, Grillari-Voglauer R et al. Differentially circulating miRNAs after recent osteoporotic fractures can influence osteogenic differentiation. Bone 201579 43-51. (doi:10.1016/j. bone.2015.05.027)

15 Chen C, Cheng P, Xie H, Zhou HD, Wu XP, Liao EY \& Luo XH. MiR503 regulates osteoclastogenesis via targeting RANK. Journal of Bone and Mineral Research 201429 338-347. (doi:10.1002/jbmr.2032)

16 Sugatani T, Vacher J \& Hruska KA. A microRNA expression signature of osteoclastogenesis. Blood 2011117 3648-3657. (doi:10.1182/blood2010-10-311415)

17 Takigawa S, Chen A, Wan Q, Na S, Sudo A, Yokota H \& Hamamura K. Role of miR-222-3p in c-Src-mediated regulation of osteoclastogenesis. International Journal of Molecular Sciences 201617 240. (doi:10.3390/ ijms17020240)

18 Brown JP, Dempster DW, Ding B, Dent-Acosta R, San Martin J, Grauer A, Wagman RB \& Zanchetta J. Bone remodeling in postmenopausal women who discontinued denosumab treatment: off-treatment 
biopsy study. Journal of Bone and Mineral Research 201126 2737-2744. (doi:10.1002/jbmr.448)

19 Brown JP, Roux C, Törring O, Ho PR, Beck Jensen JE, Gilchrist N, Recknor C, Austin M, Wang A, Grauer A et al. Discontinuation of denosumab and associated fracture incidence: analysis from the Fracture Reduction Evaluation of Denosumab in Osteoporosis Every 6 Months (FREEDOM) trial. Journal of Bone and Mineral Research 201328 746-752. (doi:10.1002/jbmr.1808)
20 Brown JP, Ferrari S, Gilchrist N, Beck Jensen JE, Pannacciulli N, Recknor C, Roux C, Smith S, Torring O, Valter I et al.

Discontinuation of denosumab and associated fracture incidence: analysis from FREEDOM and its extension. Journal of Bone and Mineral Research 201631 (Supplement 1). (Available at: http://www.asbmr.org/education/AbstractDetail?aid= 03c2777e-2cd5-4b06-8ffe-0bef664a71ee). Accessed on 7 November 2016.

Received 15 December 2016

Revised version received 5 March 2017

Accepted 10 March 2017 